\title{
A Semantic-Oriented Platform for Performance Monitoring of Distributed Java Applications
}

\author{
Włodzimierz Funika, Piotr Godowski, and Piotr Pȩgiel \\ Institute of Computer Science, AGH, al. Mickiewicza 30, 30-059 Kraków, Poland \\ funika@agh.edu.pl, \{flash,pegiel\}@student.agh.edu.pl \\ Phone: (+48 12) 61744 66; Fax: (+48 12) 6338054
}

\begin{abstract}
In this paper we present an approach to semantic performance analysis in on-line monitoring systems. We have designed a novel monitoring system which uses ontological description for all concepts exploited in the distributed systems monitoring. We introduce a complete implementation of a robust system with semantics, which is not biased to any kind of the underlying "physical" monitoring system, giving the end user the power of intelligent monitoring features like automatic metrics selection and collaborative work.
\end{abstract}

\section{Introduction}

The design of a distributed application is in many cases a challenge to the developer ([1|l|l|l|l|l $)$. On the one hand, there are the limitations and performance issues of distributed programming platforms. So one of the most important tasks is to increase the performance and reliability of distributed applications. On the other hand, the developer must assure that the application manages and uses distributed resources efficiently. Therefore, understanding application's behaviour through performance analysis and visualization is crucial. It is especially true now, when many distributed systems exploit the SOAP protocol, where functionality of the program is implemented as Web Services. The monitoring of data flow between components could be very helpful for the user to discover performance problems with a system.

The biggest problem when using performance tools (especially, these working "on-line") is their complexity. Thus many users benefit from often less complex but easier to use tools. So a very important task is to ease user's interactions with the monitoring system, moreover, to turn these interactions into a kind of collaboration activities with the system, which involve other users. Certainly, "simple" should not imply "limited functionality" related to performance evaluation. Nowadays, more and more developed software use software agents which guide the user step-by-step. Such agents usually use a semantic description of software's features and through the analysis of user's behaviour provide suggestions what to do to achieve a desired result.

A similar approach can be used in tools used for performance monitoring in the distributed environment. The first steps have been done (AutoPilot, PerfOnto),

M. Bubak et al. (Eds.): ICCS 2008, Part III, LNCS 5103, pp. 233 242, 2008.

(C) Springer-Verlag Berlin Heidelberg 2008 
but their authors aimed at developing their own architecture for semantic description (mostly based on feedback from metrics) from scratch, omitting the already existing solutions (like OWL/RDF). The Semantic Web paradigm has introduced the concept of semantic description of resources (OWL/RDF, DAML) and services (mostly Web Services - OWL-S, DAML-S). We can leverage from existing standards to develop a performance monitoring tool using some knowledge which describes performance metrics, and this is the primary goal of our paper.

The rest of this paper is organized as follows: Section 2 discusses a motivation and system use cases. Related work is discussed in Section 3, in Section 4 we present our proposed ontology and system architecture for on-line monitoring system with semantics, followed by Summary and Future work in Section 5.

\section{System Use Cases}

In this paper we are presenting a semantic-oriented monitoring infrastructure called SemMon. The architecture of the tool fits into the OMIS model [10] and is capable to co-operate with available monitoring systems (like J-OCM $[8$, JMX1]).

The complexity and heterogeneity of the technologies necessitates to introduce semantics into the distributed computing monitoring because; the large amount of hardware, software and network environments makes monitoring a challenging task. Semantics enables the system to automatically process data without supervision or customized processing for specific areas, enables "understanding" what is really monitored, which in turn reduces the time the user spends on manually searching for issues and shortens the system learning curve. Having a semantic description and taxonomy of the monitored elements and their contexts, the system is "smart" enough to guide its user throughout the whole monitoring/analysis process. The user can focus on its main task: to find performance issues within limited time, based on the system guidance coming from historic analysis and being able to add their own measurements when needed.

Semantics in the monitoring architecture should exploit as much as possible from existing solutions, libraries and tools, with special attention paid to Open Source software and solutions developed in European projects (like GOM [9] developed in the K-Wf Grid project [7]). The following general use cases show the usability of the designed system.

The user should be able to:

- monitor the performance of a Java application running under control of a physical monitoring system

- use the system in an automatic way with a set of metrics which are meaningful for the user and a desired result

- get information about metrics that should be called in a next step.

The system administrator should be able to:

- create, destroy, and insert a semantic description of available metrics and elements of the monitored system

\footnotetext{
${ }^{1}$ Stands for Java Management Extensions.
} 
- provide new metrics in a physical monitoring system, and describe them in semantic way

- manage historical performance data.

The work should be based on a portable (preferably XML) client-server protocol both for sending and receiving requests about semantic descriptions. Developing a system as a Web Service (with semantic description provided) leads to a universal solution, which fits into the Semantic Web paradigm. The developed system should be designed in such a way that it should work with any existing "native" grid-enabled monitoring system. The system should be able to integrate with existing ontologies describing resources and performance measurements which should be a great benefit for system administrators. The designed system should be able to be extended with sensors and metrics strongly related to the structure of the monitored application to point the actual and the most accurate source of the data.

\section{Related Work}

In this section we will concentrate on those available monitoring systems where semantics or flexible monitoring architecture are introduced.

Gemini [5] is a Grid monitoring framework that fulfills a gap between resources monitoring components and monitoring services clients. It performs measurements using a set of loadable modules called sensors which retrieve monitoring data on its own or by using external applications for this purpose. Although the Gemini framework is powerful in its flexibility of adding new sensors, it does not use any kind of semantics for selecting performance metrics to run and analyse or for providing any guidance to the user.

Autopilot [1] has been developed within the Grid Application Development Software (GrADS) Project [6] and is responsible for adaptive control of distributed applications. Autopilot's architecture comprises performance sensors and a decision control unit using fuzzy logic to analyse received data from sensors and preparing messages to actuators. Autopilot is the very first example of exploiting some kind of semantics usage, or rather fuzzy logic usage to help with monitoring and adaptation actions.

PerfOnto 12 is a new approach to performance analysis, data sharing and tools integration in Grids that is based on ontology. PerfOnto is an OWL ontology describing experiment-related and resource-related concepts. The experiment-related concept describes experiments and their associated performance data on applications. The prototype PerfOnto system is able to search data in an ontological (i.e. using a knowledge base) manner, e.g. to find a code region executed on a particular node with a metric exceeding a threshold value, thus giving a hint to the site scheduler to migrate a job to another node. PerfOnto gives a rich description of performance data, but does not provide any automation for using it. Whereas using much of PerfOnto's taxonomy and retaining the main idea of describing resources in form of ontology, we were able to significantly extend it and provide adaptation algorithms. 


\section{Overview of the SemMon System}

The visualization of monitoring data in a "user friendly" form is one of the most key features provided by any performance monitoring system. Due to the great amount of gathered information, proper presentation and interpretation of observation results becomes a very complicated task. So steering the visualization of monitoring data involving making decisions what, when, in what form, under which circumstances should be presented to the user is a challenge.

A high level architecture overview of SemMon is introduced in Fig. 1.

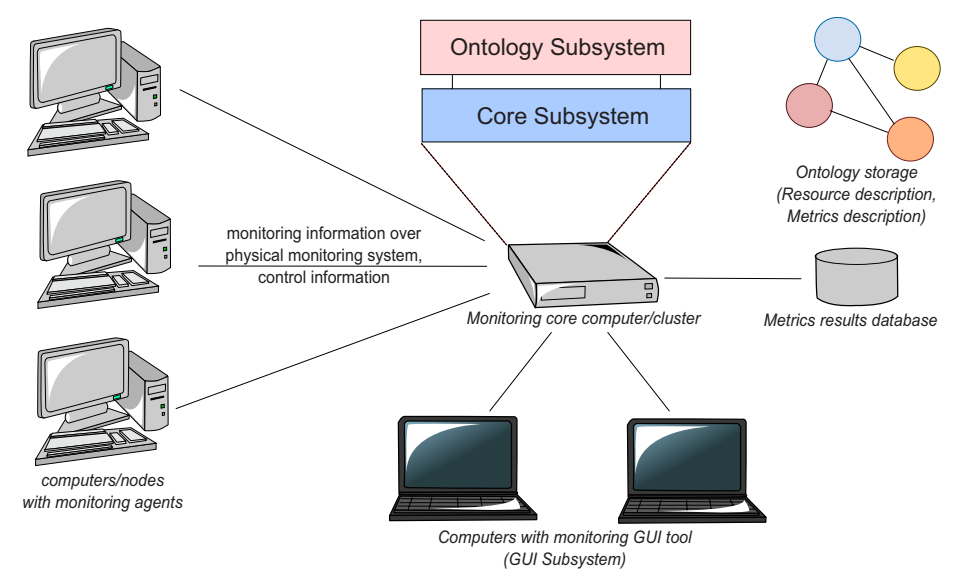

Fig. 1. System architecture as distributed environment. Monitoring core computers include Core Subsystem and Ontology Subsystem.

The heart of the model are computers that provide a primary system functionality like processing an ontology with Resource Capabilities and Metrics or storing monitoring data.

To support knowledge persistency a database is required. This functionality is implemented in the Ontology subsystem. Another part of this node is support for a "physical" monitoring system. This subsystem has to provide a functionality for registering monitoring agents as well as for processing monitoring data.

The second part of the system contains computers with monitoring agents. Agents expose monitored resources to our monitoring system. All of them will register to the Core subsystem, afterwards Core is able to introspect possible resources that are exposed. Agents are programs on the nodes/computers that access the "physical" monitoring system, e.g. JMX, JOCM.

The last part of the system are GUI clients connected to the Core subsystem. GUI is an environment for collaborative work - the users share metric ranks between different GUI instances in order to help other users in proper decision making. In the following we focus on a description of the components of the SemMon monitoring system. 


\subsection{Ontology Subsystem}

The Ontology subsystem is the heart of the whole system. The key aspect to understand here is the ontology term. An ontology is an explicit specification of a conceptualization. In such an ontology, definitions associate the names of entities (e.g., classes, relations, functions, or other objects) with a human readable text describing what the names are meant to denote, and formal axioms that constrain the interpretation and well formed use of these terms, formally specified with the OWL language. The Ontology Web Language (OWL) is intended to be used when the information needs to be processed in an automatic way by applications, as opposed to situations where the content only needs to be presented to humans. OWL has powerful facilities for expressing meaning and semantics and thus OWL goes beyond all other similar languages in its capability to represent a machine interpretable content on the Web.

The Ontology subsystem contains methods for parsing, automatic interpretation, searching, creating, and, finally, saving and sharing ontology data. The Ontology subsystem brings a unique feature to the designed system: the capability of interpreting what is monitored both for system users and (what is even more important) for the system itself. Using the knowledge deployed in the underlying ontology data, the system is aware what is monitored and what should be monitored in a next step within the monitored application's lifetime. Every single type of resource accessible to the monitoring system is described in the OWL ontology and reflects a natural computing resources hierarchy. Part of the description or even the whole of it can be updated.

Resources in question are: Resource classes (like Node, CPU, JVM), Resource instances (i.e. OWL instances of resources available in the underlying monitoring system, like CPU_i386_node2_cluster1) and the measurable attributes for the resource instances. Each Resource class defines which measurable attributes are available for its instances. A measurable attribute, called in this paper $R e$ sourceCapability, might be both an atomic attribute (like LoadAvg1Min) or an OWL superclass for a set of ResourceCapabilities. This way a natural hierarchy of capabilities can be constructed. A special property hasResourceCapability is a glue between Resources and ResourceCapabilities. Any type of Resource can contain any number of Resource Capabilities. Fig. 2 shows the Resources ontology class hierarchy while Fig. 3 presents a fragment of the ResourceCapabilities ontology class hierarchy.

An ontology describes metric concepts like the OWL classes or individuals describing metrics available to be executed by the user. Metrics ontology reflects the metrics hierarchy (i.e. from the most generic metric to the most specific one) in order to provide a rich description for ontology reasoners. Metrics can be simple, i.e. the metric is able to measure only one attribute or custom, which means that the metric can be applied to as many capabilities as required and it is even possible to provide custom implementations for metrics (user-defined metrics).

A metrics ontology is designed from a flat list of all available metrics to be considered by the monitoring system. However, having only a flat list without a hierarchy (specialization) introduced, it is impossible to provide any powerful 


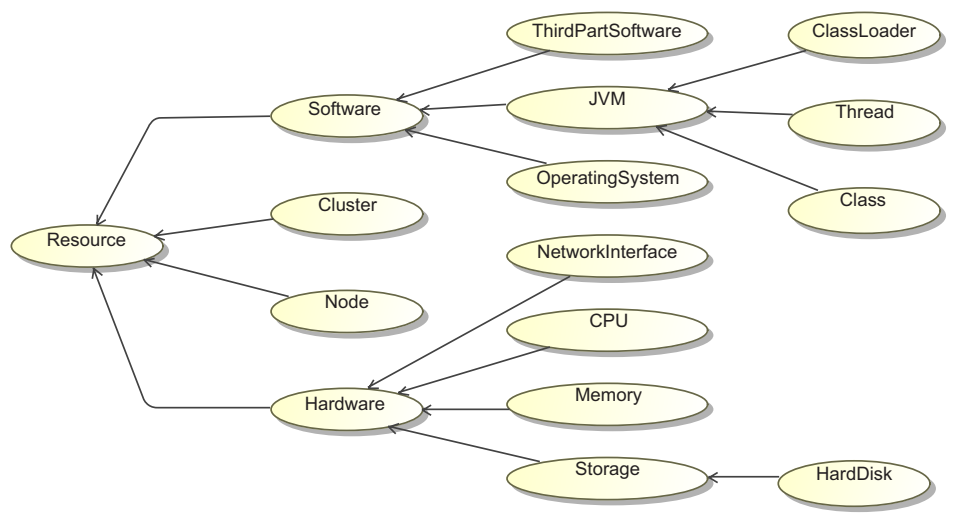

Fig. 2. Resources ontology diagram

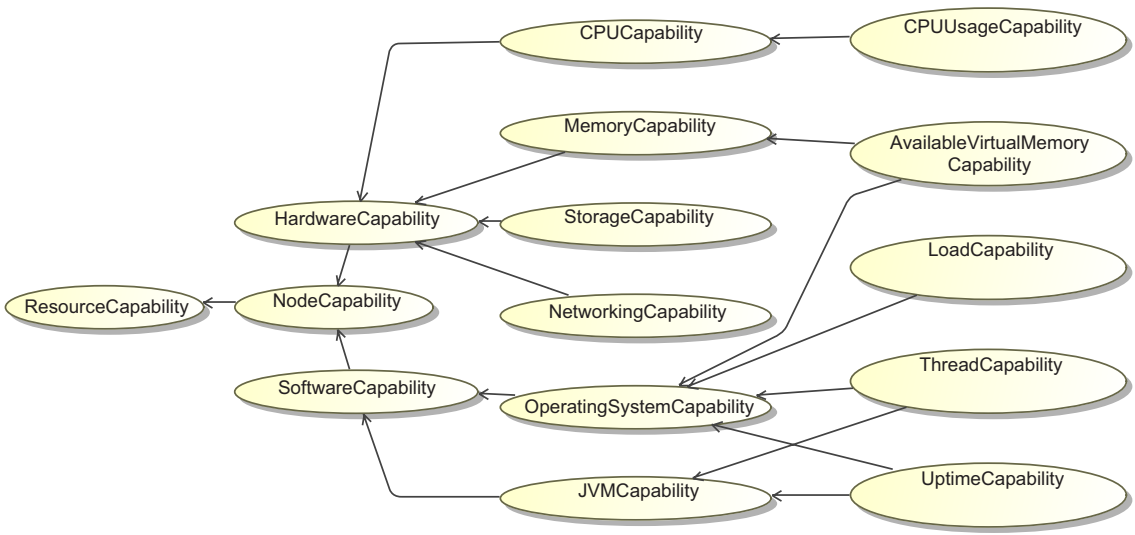

Fig. 3. ResourceCapabilities ontology diagram

reasoning process. This is because no "generic-specific" or "is related to" relationships are provided. Looking at a flat list of all possible metrics, the next step is to find out which of them are generic and which are specific. Such relationships can be expressed in an ontology as the rdfs:subClassOf property. A sample superclass metric might be SoftwareMetric with its specific subclass JVMThreadCPUTimeMetric. As a result, metrics form a tree which can be used for a reasoning process.

A special metric property monitors is a glue between the Metrics ontology and the Resources and ResourcesCapabilities ontology. Property monitors has a domain in the AbstractMetric class (and its subclasses) and a range in the ResourceCapability classes. Because the cardinality of this property is not limited, any type of AbstractMetric is able to monitor any number of capabilities. This means that the total number of measurements available in the system does not equal to the number of subclasses and individuals of the AbstractMetric 
class, but is a sum of cardinalities of monitors properties in the metrics ontology. Metric property hasCustomImplementationClass is used to inform the system that the metric is a custom metric, i.e. has its own implementation. This property points to the fully qualified Java class name implementing the Custom Metric interface. Custom Metric has its own implementation rules that is exactly returned as a measurement process, which is explained as follows. Since Custom Metric can access the Core public API, and the Resources registry, it can request any number of capabilities' values from the underlying monitoring system. The only contract that Custom Metric must meet is to return a single number each time it is requested for.

It should be noted that the described Ontology subsystem should be able to coexist with any already existing ontology for resources description and matching.

\subsection{Core Subsystem}

The Core subsystem is responsible for connecting to the underlying monitoring system's initialization (using its protocol adapter mechanism), deploying, initializing and executing metrics (including user-defined metrics), providing an interface to the Ontology subsystem and last but not least, exposing a public (remote) interface for GUI clients to connect to. Core also manages GUI clients subscribed to the list of connected resources, running metrics, running metric values and alarms (i.e. conditional action metrics notifications). The Core subsystem comprises three components - Adapter, Resource Registry, and Remote interface for GUI. The Adapter component follows the commonly used Adapter structural design pattern and is used for "translation" of all Core requests into the requests specific to the underlying monitoring system (JMX, J-OCM, OCM$\mathrm{G}$, etc.). Due to the major differences and interface incompatibilities of a wide range of monitoring systems available on the market, a common interface called Protocol Adapter is designed. Resource Registry is a service that leverages both Core and Protocol Adapter. Resource Registry holds (with Protocol Adapter) all the resource instances found as visible in the underlying monitoring system and maps them into Core identifiers. Protocol Adapter resolves incompatibility issues between different physical monitoring systems. User-defined metrics have full access to the public Core API. Therefore a user-defined metric (implementing the Custom Metric interface) can introspect Resource Registry and with a Protocol Adapter implementation is capable to send a specific query to the underlying monitoring system. This feature is useful when the underlying monitoring system has some specific features, not covered by the generic Protocol Adapter interface.

Remote interface for GUI allows remote GUI clients to connect to SemMon to enable collaborative work and provides:

- notifications for: newly attached and detached monitoring systems, started and stopped measurements on the Core subsystem, and, finally, notifications for measurement values

- interface for alarms - Alarms are conditional action metric notifications. When some action metric is running on the Core subsystem and its value ex- 
ceeds a declared threshold action value, all the unconditional action metrics that are declared in the underlying ontology are sent as notifications to all the subscribed GUI users. The user is enabled to take an action to resolve the alarm (e.g. to start a new metric from within a list of metrics suggested by the system).

\subsection{Sample Use of SemMon}

The below real-life example shows a few of the key SemMon features. A SemMon system user is monitoring a complex distributed application with critical problems relating to unstable memory usage over the application life time occurring only on a single node of the cluster. The monitored application is a WebServiceenabled Java server, deployed across a cluster of processing nodes placed behind a restrictive firewall with a load balancer. The usual behaviour on the correctly deployed system in question is to consume $50 \%$ of the CPU time for each node and create no more than 100 threads per JVM instance.
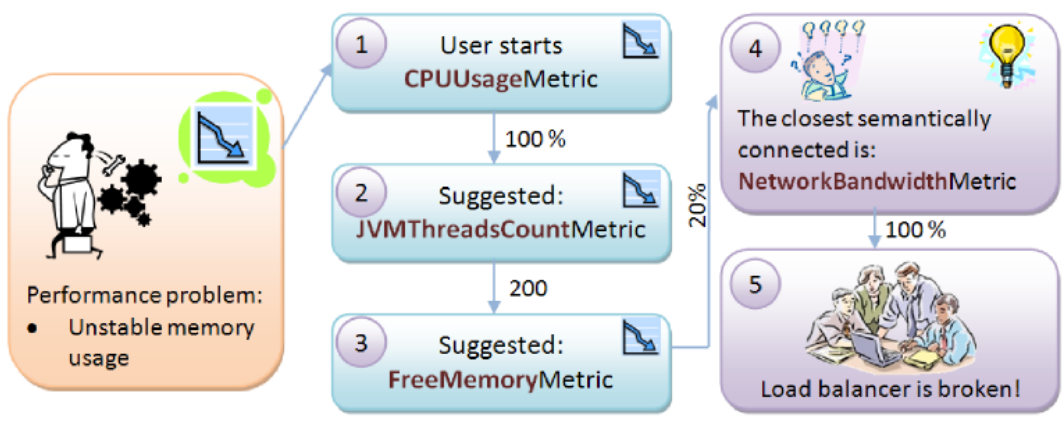

Fig. 4. Sample analysis with SemMon

At first, the user decides to monitor a CPU usage on the heaviest loaded node (see step 1 in Fig. 4). When a CPU burst lasts for at least 3 minutes, SemMon deducts from the metrics ontology a "critical" situation, and calculates the next most probable metric to start. Since CPU load is semantically connected with the number of JVM live threads, the adequate metric is suggested and the user follows this guidance (2). Again, the number of threads is irrationally high (over 200 threads), so a new alarm is raised and SemMon "reasons" that since the CPU load and number of threads were already monitored, it would be reasonable to monitor memory usage (3). Since the memory usage is at $80 \%$ level of the available virtual memory, a new alarm is raised. This moment is the key in the described monitoring scenario: since the observed CPU load, memory usage, and JVM threads count are extremely high, the algorithm selects the Network Bandwidth metric to run (4). A motivation for doing this is that this metric had been frequently selected by other system users in the past and it is semantically connected with memory usage, CPU load, and JVM threads (there 
is a possibility that application threads are processing data incoming from the network). SemMon has calculated the best matching metric and the user is able to see that almost the whole available network bandwidth is consumed by the incoming traffic. This suggests that either the cluster system is overloaded (which is not the case since we observe high load only on the certain node), or the load balancer is broken. The user checks the network bandwidth on the rest of the cluster nodes and does not observe any significant incoming traffic. This leads to the conclusion that the problem lies not in the monitored application, but in the load balancing component (5).

Please note that the path followed by the user comprises both hardware (low level) and software (high level) metrics. It shows how flexible a reasoning process might be when the knowledge stored in SemMon holds possibly a full description of the environment. It is also possible to track down performance issues not only in the monitored application, but also in its environment.

\section{$5 \quad$ Summary and Future Work}

The main objective of this paper was to present the design and implementation of a robust and flexible semantics-oriented monitoring system, SemMon. It seems to be one of the first complete approaches to the joint "worlds" of on-line distributed monitoring and Semantic Web.

The SemMon system extensively uses ontology for semantic description of all concepts used in. It is as much flexible as it can be, starting from picking up automatic ontology changes, through automatic metric selection assistance, collaborative users' knowledge leveraging, user-defined metrics, finally, to the extensible and clear visualisation options.

There are still places for improvements. There is a unresolved problem with performing part of the computations on the clients to improve system scalability by reducing the size of performance data sent to a central database. An important task is to explore algorithms for reasoning in the ontology frameworks. Although there are some improvements in the query algorithms, they are just based on additional caching layer rather than optimizing algorithms.

Acknowledgements. The research is partially supported by the EU IST 0004265 CoreGRID and 031857 int .eu.grid projects with the related SPUB-M grant.

\section{References}

1. Gerndt, M., Wismüller, R., Balaton, Z., Gombás, G., Kacsuk, P., Németh, Zs., Podhorszki, N., Truong, H.-L., Fahringer, T., Bubak, M., Laure, E., Margalef, T.: Performance Tools for the Grid: State of the Art and Future. APART-2 Working Group, Research Report Series, Lehrstuhl für Rechnertechnik und Rechnerorganisation (LRR-TUM) Technische Universität Muenchen, vol. 30. Shaker Verlag (2004) ISBN 3-8322-2413-0

2. Podhorszki, N., Kacsuk, P.: Presentation and Analysis of Grid Performance Data. In: Kosch, H., Böszörményi, L., Hellwagner, H. (eds.) Euro-Par 2003. LNCS, vol. 2790, pp. 119-126. Springer, Heidelberg (2003) 
3. Reed, D.A., Ribler, R.L.: Performance Analysis and Visualization. In: Foster, I., Kesselman, C. (eds.) Computational Grids: State of the Art and Future Directions in High-Performance Distributed Computing, pp. 367-393. Morgan-Kaufman Publishers, San Francisco (1998)

4. Wismüller, R., Bubak, M., Funika, W.: High-level application-specific performance analysis using the G-PM tool. Future Generation Comp. Syst. 24(2), 121-132 (2008)

5. Balis, B., Bubak, M., Labno, B.: GEMINI: Generic Monitoring Infrastructure for Grid Resources and Applications. In: Bubak, M., Unger, S. (eds.). Proc. Cracow Grid Workshop 2006. The Knowledge-based Workflow System for Grid Applications, pp. 60-73. ACC Cyfronet AGH, Poland (2007)

6. Berman, F., Chien, A., Cooper, K., Dongarra, J., Foster, I., Johnsson, L., Gannon, D., Kennedy, K., Kesselman, C., Reed, D., Torczon, L., Wolski, R.: The GrAds project: Software support for high-level grid application development. Technical Report Rice COMPTR00-355, Rice University (2000)

7. Bubak, M., Fahringer, T., Hluchy, L., Hoheisel, A., Kitowski, J., Unger, S., Viano, G., Votis, K., K-WfGrid Consortium: K-Wf Grid Knowledge based Workflow system for Grid Applications. In: Proc. Cracow Grid Workshop 2004, p.39. ACC CYFRONET AGH, Poland (2005) ISBN 83-915141-4-5

8. Funika, W., Bubak, M., Smętek, M., Wismüller, R.: An OMIS-based Approach to Monitoring Distributed Java Applications. In: Kwong, Y.C. (ed.) Annual Review of Scalable Computing, ch.1, vol. 6, pp. 1-29. World Scientific Publishing Co. and Singapore University Press, Singapore (2004)

9. Krawczyk, K., Slota, R., Majewska, M., Kryza, B., Kitowski, J.: Grid Organization Memory for Knowledge Management for Grid Environment. In: Proc. Cracow Grid Workshop 2004, pp.109-115. ACC CYFRONET AGH, Krakow, Poland (2005) ISBN 83-915141-4-5

10. Ludwig, T., Wismüller, R., Sunderam, V., Bode, A.: OMIS - On-line Monitoring Interface Specification (Version 2.0). LRR-TUM Research Report Series, vol. 9. Shaker Verlag, Aachen (1997)

11. Ribler, R.L., Vetter, J.S., Simitci, H., Reed, D.A.: Autopilot: Adaptive Control of Distributed Applications. In: Proc. 7th IEEE High-Performance Distributed Computing Conference (1998)

12. Truong, H.-L., Dustdar, S., Fahringer, T.: Performance metrics and ontologies for Grid workflows. Future Generation Comp. Syst. 23(6), 760-772 (2007) 